\title{
Über die Bettruhe
}

\section{Eberhard Wolff}

Prof. Dr. rer. soc., Redaktor Kultur, Geschichte, Gesellschaft

Heute stellt die «Bettruhe» eher ein medizinisches Problem als eine therapeutische Option dar. Vor gut hundert Jahren war das ganz anders. Das Auf und Ab hat mehr als nur medizinische Gründe.

«Strenge Bettruhe!» Bei diesen Worten erscheint vor meinem geistigen Auge ein respektheischender graumelierter Herr Doktor im weissen Kittel, der sie mit ernstem Blick «verordnet». Am ehesten in einem alten Schwarz-Weiss-Film. In der Tat lag die grosse Zeit der "Bettruhe» in den Jahrzehnten, in denen Filme schwarz-weiss und Ärzte männliche Autoritäten waren.

Bettruhe gibt es als Praxis, solange es Betten gibt. Wer krank und schwach ist oder nicht aufstehen kann, liegt im Bett. Und seit der Antike wurden Ruhe und Bewegung als Faktoren eines gesunden Lebenswandels angesehen. Auch die geregelte Bettruhe von Wöchnerinnen stellt eine traditionelle Praxis dar. Als verbreitetes ärztlich-therapeutisches Konzept bei Krankheit ist die «Bettruhe» jedoch ein Kind des späten 19. Jahrhunderts.

$\mathrm{Zu}$ den frühesten Vertretern einer therapeutischen Ruhe bzw. Erholung zählte der Londoner Anatom und Chirurg John Hilton in seinem Buch On Rest and Pain von 1862. In der Folge verordneten niedergelassene und Spitalärzte die «Bettruhe» immer häufiger als uni-

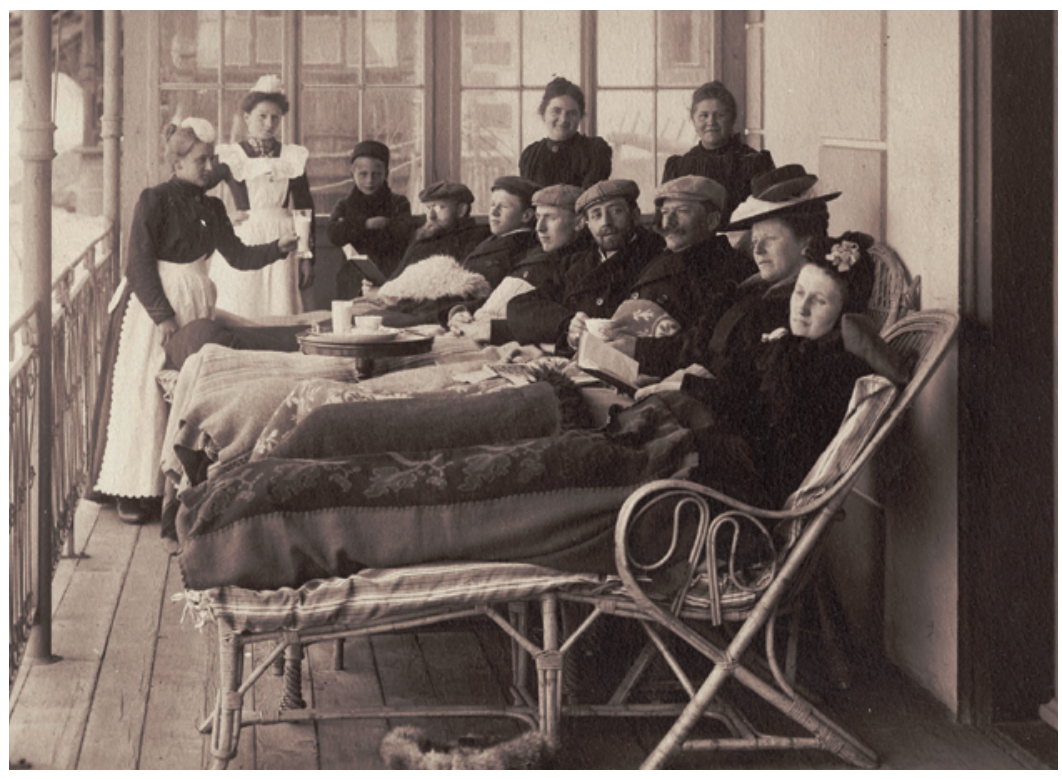

Liegekur auf der Terrasse der Villa Pravenda in Davos (um 1900). versell eingesetzte Form der Krankheitsbehandlung. In der deutschsprachigen Literatur schoss die Verwendung des Begriffs in den 1880ern steil in die Höhe [1].

\section{"Liegekuren" in den Bergen - «Bettbehandlung» in der Psychiatrie}

Die heute noch bekannteste therapeutische Bettruhe betrieben die damaligen Höhensanatorien zur Behandlung der grassierenden Tuberkulose. Bei der «Höhenkur» täglich stundenlang im Liegestuhl oder Bett an der frischen Luft zu liegen war eben nicht nur als Luftkur und Lichttherapie gedacht, sondern gleichzeitig auch als Liegekur, bei der es um therapeutische Schonung ging - um "Kräfteersparnis», wie es in Thomas Manns Zauberberg von 1924 heisst.

Die Liegekuren fielen zusammen mit dem sogenannten «nervösen Zeitalter» der Hochindustrialisierung und Lebensbeschleunigung. Die Ärzteschaft erklärte Krankheiten damals sehr gerne mit der Modediagnose «Neurasthenie» als Schwächezustand der Überreizung und Erschöpfung. So war die Medizin eben auch eng mit dem «Zeitgeist» verbunden.

War die Bettruhe selbst gewollt, konnte sie ein Prestigeobjekt für die Patientinnen und Patienten sein Man zeigte, dass man sie sich leisten konnte. Anders in den «Volkssanatorien». Hier war die extensive Liegekur mehr ein Akt der Disziplinierung [2].

Auch in der Psychiatrie wurde die Bettruhe als tageund wochenlange «Bettbehandlung» um 1900 zu einer Art Allheilmittel. Sie sollte die Kranken nicht einfach nur beruhigen, sondern genauso unter Kontrolle halten. Das Liegen im Bett konnte den Uneinsichtigen zudem praktisch deutlich machen, dass sie krank waren [3].

\section{Rebellionen gegen die Bettruhe}

Bettruhe war oft mit Machtstrukturen verbunden. Joseph Roth beschreibt in seinem Roman Rebellion (1924) etwa die Behandlung kranker Zuchthäusler: «dann liegt man mit achtzig geschwollenen Männern in einem Saal und lässt sich durch Bettruhe kurieren. Manchmal stirbt man ein wenig an der Bettruhe» [4]. Selbst die private Bettruhe zu Hause bedeutete in der zeitgenössischen Literatur ein Stück Machtausübung, 
nicht zuletzt über Frauen. In der Kurzgeschichte The Yellow Wallpaper hielt die amerikanische Schriftstellerin Charlotte Perkins Gilman 1892 den Horror fest, durch eine von ihrem Arzt-Ehemann verschriebene "Ruhekur» zum Nichtstun gezwungen zu sein. Im richtigen Leben war der Hausarzt der Autorin, Silas Weir Mitchell, wirklich ein bekannter Vertreter einer solchen "Rest Cure». Die Kurzgeschichte entwickelte sich zu einem Referenztext des frühen Feminismus.

Selbst Virginia Woolf machte sich 1925 in Mrs. Dalloway über die Bettruhe als Therapie der «Nervenkrankheiten" lustig: "Ruhe in Einsamkeit; Stille und Ruhe; Ruhe ohne Freunde, ohne Bücher, ohne Botschaften; sechs Monate Ruhe; bis ein Mann, der beim Hineingehen gut hundert Pfund wog, herauskommt und hundertsiebzig wiegt [5].

Während der Pandemie der Spanischen Grippe ab 1918 spielte die Bettruhe mangels einer effizienten Therapie eher die Rolle einer mehr oder weniger hilflosen Ersatzbehandlung.

\section{Der Niedergang}

Der Höhenflug der Bettruhe dauerte nur wenige Jahrzehnte. In den Psychiatrien ersetzte schon ab den 1920er Jahren die rentablere "Arbeitstherapie» die Bettbehandlung - auch angesichts von Krieg und Krise [3]. Die tuberkulösen Sanatoriumspatienten auf den sonnendurchfluteten Balkonen in den Bergen arbeiteten oder lernten nun ebenfalls immer häufiger in ihren Betten und machten sich damit "nützlich», noch bevor das Streptomycin sie schneller aus der Liegekur trieb.

Konkretere medizinische Bedenken gegenüber der Bettruhe brachte die beginnende US-Raumfahrt-Forschung ab den 1950ern hervor. Ein längeres passives Liegen habe nachteilige Auswirkungen, etwa auf den Kreislauf oder das Muskuloskelettalsystem [6]. Probleme wie das Thromboserisiko oder die Muskelatrophie traten nun immer stärker in den Fokus unterschiedlichster Studien. In den Spitälern holte man die frisch Operierten jetzt so schnell es ging für Gehübungen wieder aus den Betten. Die Bettruhe änderte in der Spitalversorgung ihren dominanten Charakter von «Therapie» zu «Problemfall». «Mobilisation» wurde zum neuen Zauberwort [7, 8].

Der Bettruhe blieben einige Rückzugsgebiete, z.B. als niederschwellige Standardempfehlung in der Allge- meinpraxis [9] oder als Burnout-Begleittherapie. Auch die Langzeitnarkose (vulgo: künstliches Koma) enthält Komponenten der therapeutischen Bettruhe.

\section{Aktivität und Effizienz statt Bettruhe}

Auch der Übergang vom Zeitalter der Bettruhe in das der Patientenmobilisation dürfte mit Entwicklungen jenseits von Therapie, ja Medizin überhaupt, zusammenhängen: mit der Dominanz des Aktivitäts- und Effizienzdenkens, in der die Klinikaufenthalte aus ökonomischen Gründen immer kürzer werden; mit einer Zeit, in der Patienten immer weniger als passive Empfänger medizinischer Wohltaten verstanden werden, sondern als aktive, immer eigenverantwortlichere Gesundheitsakteure; mit einer Zeit, in der nicht nur die Grenzen zwischen Gesundheit und Krankheit verschwimmen. Das passive Modell der Bettruhe passt nicht mehr in unser Zeitalter.

Das habe ich kürzlich selbst erlebt. Mich hatte ein Infekt für ein, zwei Tage ins Bett geworfen. Bei etwas über 38 Grad Fieber erledigte ich mit dem Laptop auf der Bettdecke aber noch ein paar wichtigere Mailgeschäfte. Die Antwort eines Kollegen kam ziemlich schnell - mit besten Grüssen aus einem Spitalbett. Der graumelierte Herr Doktor, der «strenge Bettruhe» einfordert, hat ausgedient.

Literatur

1 Das zeigt eine Recherche des prozentualen Auftretens des Begriffs mit dem «Ngram Viewer»: https://books.google.com/ngrams Etwas später, aber ähnlich die Begriffe «Bettbehandlung» und "rest cure».

2 Ritzmann I. Hausordnung und Liegekur. Vom Volkssanatorium zur Spezialklinik: 100 Jahre Zürcher Höhenklinik Wald. Zürich: Chronos; 1998. Schürer C. Der Traum von Heilung. Eine Geschichte der Höhenkur zur Behandlung der Lungentuberkulose. Baden Hier und Jetzt; 2017.

3 Ankele M. Horizontale Szenographien. Das Krankenbett als Schau platz psychiatrischer Subjektivation. In: Friedrich L, Harrasser K, Kaiser C (Eds.). Szenographien des Subjekts. Wiesbaden: Springer; 2018 (49-64).

4 Roth J. Die Rebellion. Göttingen: Wallstein; 2019 (o. S)

5 Woolf V. Mrs Dalloway. München: Anaconda; 2013 (207).

6 Sprague AE. The evolution of bed rest as a clinical intervention. J Obstet Gynecol Neonatal Nurs. 2004 Sep-Oct;33(5):542-9.

7 Für die SÄZ als neueres Beispiel: Suhm N, Saxer F. Junge Alterstraumatologie nützt älteren Unfallpatienten! Schweiz Ärzteztg. 2017;98(47):156-7.

8 Für das SMF als neueres Beispiel: Méan M, Bula C, Waeber G. Bettruhe während des Spitalaufenthalts. Swiss Medical Forum. 2017;17(7):152-4.

9 Schreier M. Mein letzter Notfalldienst. Schweiz Ärzteztg. 2003;84(1-2):40-1.

Bildnachweis

() Dokumentationsbibliothek Davos 